\title{
CHARACTERIZATION APPROACHES TO PLACE INVARIANT SITES ON SI- TRACEABLE SCALES
}

\author{
K. Thome
}

\section{NASA/GSFC}

\section{INTRODUCTION}

The effort to understand the Earth's climate system requires a complete integration of remote sensing imager data across time and multiple countries. Such an integration necessarily requires ensuring inter-consistency between multiple sensors to create the data sets needed to understand the climate system. Past efforts at inter-consistency have forced agreement between two sensors using sources that are viewed by both sensors at nearly the same time, and thus tend to be near polar regions over snow and ice. The current work describes a method that would provide an absolute radiometric calibration of a sensor rather than an inter-consistency of a sensor relative to another. The approach also relies on defensible error budgets that eventually provides a cross comparison of sensors without systematic errors. The basis of the technique is a model-based, SI-traceable prediction of atsensor radiance over selected sites. The predicted radiance would be valid for arbitrary view and illumination angles and for any date of interest that is dominated by clear-sky conditions. The effort effectively works to characterize the sites as sources with known top-of-atmosphere radiance allowing accurate intercomparison of sensor data that without the need for coincident views.

Development of methods that do not require coincident views would greatly increase the possible number of cross-comparison opportunities as well as permit the use of alternate surface types. Such approaches must overcome issues from changes in illumination and view conditions as well as physical changes in the atmosphere and surface over time. Teillet et al. developed an approach to overcome small differences in view and solar geometry relying on an aircraft sensor to derive the surface reflectance of a test site both spatially and spectrally. ${ }^{1}$ The derived surface reflectance is an input to a radiative transfer code, along with the coincident atmospheric data which permits prediction of the at-sensor radiance. Such an approach was used to cross-compare data from a wide array of sensors viewing the Railroad Valley test site on a single day but at varying times and with varying view angles. ${ }^{2}$ 
Improved precision of the reflectance-based approach and vicarious approaches in general have allowed their results to be used as the cross-comparison approach in the same fashion as preflight, laboratory calibrations allow intercomparisons of sensor data. The basic concept is that the radiance from a well-characterized test site can be predicted for a given sun-sensor geometry. The characterization can be based on a model-centric approach such as that developed for the comparison of AVHRR sensors over time based on desert scene data. ${ }^{3}$ Alternatively, the characterization can rely on in-situ measurements at the time of both sensors, in which case the in-situ measurements themselves act as the transfer standard. ${ }^{4}$

Data from the Advanced Spaceborne Thermal Emission and Reflection and Radiometer (ASTER), Enhanced Thematic Mapper Plus (ETM+), and Moderate Resolution Imaging Spectroradiometer (MODIS) are used to demonstrate the difficulties of cross calibration as applied to current sensors. Special attention is given to the differences caused in the cross-comparison of sensors in radiance space as opposed to reflectance space. The radiance comparisons lead to significant differences created by the specific solar model used for each sensor. The paper also proposes methods to mitigate the largest error sources in future systems.

The results from these historical intercomparisons provide the basis for a set of recommendations to ensure future SI-traceable cross calibration using future missions such as CLARREO and TRUTHS. The paper describes a proposed approach that relies on model-based, SI-traceable predictions of at-sensor radiance over selected sites. The predicted radiance would be valid for arbitrary view and illumination angles and for any date of interest that is dominated by clear-sky conditions. The basis of the method is highly accurate measurements of at-sensor radiance of sufficient quality to understand the spectral and BRDF characteristics of the site and sufficient historical data to develop an understanding of temporal effects from changing surface and atmospheric conditions.

\section{COINCIDENT VIEW RESULTS}

The most cross-calibration approach is one in which the sensors view the same area with the same view angle at the same time. Ideally, the spectral bands would also be identical. The ASTER/MODIS intercomparison case satisfies all but the last, in that while several bands are similar between the sensors, they are not identical. Results from ASTER and MODIS show a degradation in ASTER data that is verified through other approaches but the scatter in the cross-comparisons due to errors in 1) the spectral correction; 2) registration of the common areas used for the cross-calibration; and 3) temporal variability in the sensors relative to one another are clearly present. The dominant error is the registration between the two data sets with the large footprint size of the MODIS sensor causing most of the difficulties. Such an error is reduced when larger, more homogeneous areas 
are used but comparison of results from multiple test sites show a bias caused by the spectral differences between ASTER and MODIS not being properly corrected due to lack of knowledge of the spectral reflectance of the surface. Thus, the key characterization needed is a spectral knowledge of the test site.

\section{MODEL-BASED CALIBRATION APPROACHES}

The basic philosophy of the model-based calibration approach is adequate prediction of at-sensor radiance for arbitrary altitudes, view-sun geometries, and ranges of spatial resolutions. The basis of the predictions are surface and atmospheric characterization data based on historical archives of imagery for a given test site. The initial method proposed here is the development of a hyperspectral BRDF model of the surface and a set of average atmospheric conditions. This would allow a user to select a view angle and azimuth and sensor altitude as well as a set of spectral bands. A look-up table approach would supply the user with an image at a spatial resolution of 30-m over an area of $20 \mathrm{~km}$ by $20 \mathrm{~km}$ sufficient to allow intercomparisons between a wide range of sensors.

\section{SI TRACEABILITY}

The key element missing in current approaches using desert sites as invariant scenes is the lack of SI traceability. These methods are only useful in cases where there is overlap in sensor operation. Note, there is not a requirement in coincident views, and as such this is a vast improvement over methods developed as recently as 10

years ago. The difficulty occurs when there are gaps in the data records between sensors. Creating an SItraceable at-sensor radiance would solve these issues while raising a set of added issues/uncertainties. It should be kept in mind that a true SI-traceable method would include the impact of uncertainties due to atmospheric effects and surface BRDF within a realistic error budget. Inclusion of data from sensors such as CLARREO which will have vastly improved absolute accuracies over current systems will permit enhanced understanding of the test sites. The higher accuracy and spectral sampling will allow separation of surface effects from atmospheric effects permitting the development of the needed models for the at-sensor radiance prediction.

\section{CONCLUSIONS}

The exciting prospect for cross-calibration methods is that they have, in general, improved dramatically in recent years. The improvement has been both in precision and accuracy. Much of the improvement has been obtained by working in reflectance rather than radiance reducing uncertainties caused by differences in solar zenith angles. Further improvements have been obtained through the collection and inclusion of hyperspectral surface reflectance data to reduce the effects of spectral differences. Likewise, efforts to operate sensors with a 
consistent solar model have helped. The solar irradiance case is an excellent example of multi-national groups working within agencies such as the Committee on Earth Observing Satellites and the Global Earth Observing System of Systems to develop the best set of results. It will be through these international collaborations and the advent of high-accuracy sensors such as CLARREO that will permit the future improvement of imaging sensors to permit development of climate quality data records.

\section{REFERENCES}

1. Teillet, P. M., G. Fedosejevs, R. P. Gauthier, N. T. O’Neill, K. J. Thome, S. F. Biggar, H. Ripley, and A. Meygret, “A generalized approach to the vicarious calibration of multiple Earth observation sensors using hyperspectral data," Remote Sensing of Environment, 77, pp. 304-327, 2001.

2. Thome K. J., S. F. Biggar, W. T. Wisniewski, "Cross-comparison of EO-1 sensors and other Earth Resources Sensors to Landsat7 ETM+ Using Railroad Valley Playa,” IEEE Trans. On Geosciences and Remote Sensing, Vol. 41, pp. 1180-1188, 2003.

3. E. F. Vermote, and N. Z. Saleous, "Calibration of NOAA-16 AVHRR over a desert site using MODIS data," Remote Sensing of Environment, Vol. 105, pp. 214-220, 2006.

4. $\quad$ Thome, K. J., "In-flight intersensor radiometric calibration using vicarious approaches,” Post-Launch Calibration of Satellite Sensors, Edited by S. A. Morain and A. M. Budge, Balkema Publishers, Philadephia, pp. 93-102, 2004.

Verify Data

Your paper number is: 4906

Your paper access password is: EDDBF9CD

Paper Title: CHARACTERIZATION APPROACHES TO PLACE INVARIANT SITES ON SITRACEABLE SCALES

Primary Session Category: I.29 - Invited Sessions: In Flight Calibration of Optical Satellite Sensors Using Pseudo Invariant Calibration Sites

Secondary Session Category: (No secondary topic selected.)

Tertiary Session Category: (No tertiary topic selected.)

Authors:

1. Kurtis Thome, NASA/GSFC (*) 